\title{
Laevinus Torrentius et le tyrannicide
}

Marie Delcourt

\section{Citer ce document / Cite this document :}

Delcourt Marie. Laevinus Torrentius et le tyrannicide. In: Revue belge de philologie et d'histoire, tome 30, fasc. 1-2, 1952. pp. 169-174;

doi : 10.3406/rbph.1952.2134

http://www.persee.fr/doc/rbph_0035-0818_1952_num_30_1_2134

Document généré le 28/06/2017 


\section{LAEVINUS TORRENTIUS ET LE TYRANNICIDE}

En 1582, lorsque le Basque Juan Jauréguy tenta d'assassiner à Anvers Guillaume d'Orange, Torrentius, vicaire-général du prince-évêque de Liège, écrivit à la louange du meurtrier un poème qui n'a pas été conservé. Deux ans plus tard, lorsqu'à Delft Balthasard Gérard fit le même attentat et cette fois avec succès, Torrentius écrivit un nouveau poème, où il reprit probablement les thèmes du premier, ce qui expliquerait qu'il n'a laissé subsister que le second dans l'ćdition complète des Poemata Sacra de 1594. Grande fut sa complaisance envers cet écrit dont il parla beaucoup et dont il envoya copie à ses amis (1).

Ses biographes n'ont point partagé cette indulgence. Ils ont unanimement déploré qu'un prélat plein d'humanité se fût laissé égarer par le fanatisme au point de faire l'éloge d'un assassin. A. Roersch, dans sa note de la Bibliotheca Belgica, rappelle la phrase des Goncourt : "La théorie du tyrannicide? Elle est chantée dans les poésies sacrées de Livinus Torrentius, évêque d'Anvers" ( $\left.{ }^{2}\right)$, et la remarque d'Eugène Hubert, "que des écrits comme celui de Torrentius firent une impression détestable sur les protestants des Provinces-Unies lesquels redoublèrent, d'ailleurs, de sévérité à l'égard des catholiques après l'assassinat du prince d'Orange et répandirent dans le public la traduction du poème, afin de pousser davantage à la haine contre l'Espagne " $\left(^{2}\right)$.

Qu'ils aient utilisé le poème pour leur propagande, c'était de bonne guerre. Mais leur indignation était factice. Tout le monde, à cette époque, admettait le meurtre politique. Grégoire XIII fit célébrer un Te Deum après la St-Barthélemy.

(1) Corresp. de Torrentius, éd. par M. Delcourt et J. Hoyoux, T. I, I. 93, 102, T. II, L. 612.

(2) Bibl. Belg., $3^{\text {e }}$ série, s. v. Les Goncourt mentionnent le poème dans leur livre En 18... écrit en 1851,imprimé à Bruxelles en 1884. Il est très curieux qu'ils aient eu connaissance de cette œuvrette oubliée, assez difficile à trouver. 
Des historiens catholiques ont soutenu que ces actions de grâces visaient uniquement le salut du roi menacé par les huguenots. Pastor reconnaît que leur thèse est insoutenable, mais tient à dire que Grégoire XIII n'a rien su du projet: personne en 1572 ne l'en aurait cependant blâmé, ainsi que le prouvent les reproches du secrétaire d'État Galli au nonce Salviati, d'avoir été au courant du plan et de ne lui en avoir rien dit $\left(^{1}\right)$. Un homme excommunié par le pape était hors la loi : on pouvait l'assassiner sans pécher. Les excommunications de Pie V, puis de Grégoire XIII, contre Élisabeth eurent pour effet immédiat de provoquer des attentats. Le cardinal Galli, pour lequel Torrentius avait la plus vive admiration, avait très imprudemment remis à $W$. Parry une lettre d'encouragement dont celui-ci fit état après l'échec de sa tentative contre la reine $\left(^{2}\right)$. Le prince d'Orange n'avait pas été excommunié, mais seulement banni par le roi catholique. Dans l'esprit des contemporains, les deux mesures n'avaient ni un caractère, ni des conséquences très différentes. "Orangii foeda atque inopinata neque a rege, quamvis jure id fieri potuit, illata mors", écrit Torrentius à Ernest de Bavière le 28 juill. 1584. Le P. Ém. Michael, répondant à W. Ochsli qui attribuait globalement aux Jésuites $\mathrm{du}_{\mathrm{xvI}}{ }^{\mathrm{e}} \mathrm{s}$. une doctrine favorable au tyrannicide, essaie de prouver que Mariana, qui en effet la soutient dans son De rege et regis institutione (1599), est un isolé $\left({ }^{3}\right)$. Tous les événements contemporains lui donnent un démenti et Echsli aurait pu utiliser contre lui le poème de Torrentius ainsi que pas mal de textes, notamment du doux Mélanchton, qui prouvent que les Jésuites, eussent-ils unaniment approuvé le tyrannicide, n'auraient pas eu grand chose à se reprocher. Georges Weill a bien montré que, pendant les guerres de religion, chaque parti a-accordé les béné-

(1) PAstor, Gesch. der Päpste, IX, p. 369, n. 3. Je renvoie au texte allemand, la traduction française étant un tissu d'erreurs et de contre-sens.

(2) Ibid., p. 320-332. Sur les sentiments de Sixte $V$ au moment de l'assassinat de Henri III, cf. PASTOR, t. X, p. 236 et la curieuse note 4.

(3) Zeitschrift F. Theologie, 1892, p. 556 sqq. 
fices résultant du droit divin au souverain qui le favorisait, pour les lui refuser s'il adhérait à la confession opposée (1).

On pourrait donc s'attendre à trouver dans le poème de Torrentius l'expression du fanatisme religieux. Il n'en est rien. Sur les 37 strophes alcaïques qui le composent, trois seulement font allusion au caractère doctrinal de la révolte représentée par Guillaume :

Cur templa Divum dejicit ardua, Evertit aras, frangit imagines

Ac signa, quae nos usque nostrae

Esse jubent memores salutis?

Pejora dicam, et quae magis attinent.

Cur damnat illam quam magis colimus crucem, Mortisque devictae tropaeum

Mente mala furiatus odit?

Est his quod addam, dum sceleri scelus

Innectit. Ipsum cur adytis deum

Sub pane sacrato latentem

Vitigeno et medium liquore

Exturbat amens?

Le reste montre le prince déchaînant la guerre sur le pays par ambition coupable et le soulevant contre son roi légitime, et se termine par un dithyrambe de 19 strophes sur l'héroïsme de Gérard vainqueur du tyran :

Quid tam notandum praestitit Hercules?

Majore quam portenta fructu

Mortiferi pereunt tyranni.

Cet accent antique nous invite à revenir au texte contemporain qui donne la théorie du meurtre politique. Étienne de la Boétie mourut en 1563 ; la Servitude volontaire circula en manuscrit, puis fut imprimée par Simon Goulart dans les $M \dot{e}-$ moires de l'État de France sous Charles IX qui eurent trois

(1) Les théories du pouvoir royal en France pendant les guerres de religion, Paris, thèse, 1892, notamment pp. 69 et 85, qui renvoic à Bodin, Républ. II, 5. Pour une période plus ancienne, cf. Friedrich Schofnstedt, Studien zum Begriff des Tyrannen und zum Problem des Tyrannenmordes im Spätmittelalter, Thèse de Würzbourg, 1938. 
éditions de 1576 à 1578. L'éloge qu'elle fait des tyrannicides donne exactement le thème sur lequel Torrentius a brodé :

«Brute le Jeune et Casse òtèrent bien heureusement la servitude : mais, en ramenant la liberté, ils moururent, non pas misérablement, car quel blasphème serait-ce de dire qu'il y ait rien eu de misérable en ces gens-là, ni en leur mort ni en leur vie..."

Or Montaigne, lui aussi, fait l'éloge de Balthasard Gérard et c'est au chapitre de la Vertu (II, 29). Lui si parfaitement libre de tout fanatisme politique et religieux, lui qui n'avait aucune raison d'aimer Philippe II ni de souhaiter du mal à Guillaume d'Orange, il parle de Gérard avec une admiration dont il dit les raisons: que l'échec de Jauréguy, loin de le détourner, l' échauffa"; qu'il agit sans aucun espoir d'échapper à la mort; qu'il l'affronta avec un calme parfait: "Quand on lui prononça son horrible sentence: J'y étais préparé, dit-il ; je vous étonnerai de ma patience."

\section{At tu irretorta singula perferens \\ Cervice, mentemque indomitam gerens \\ Non voce testatus dolorem, \\ Non gemitu, sed in astra vultus \\ Fingens viriles, nec timidus mori...}

A l'acte délibéré de Gérard, Montaigne oppose l'assassinat du duc de Guise par Poltrot, où "il y eut plus de hasard que de vigueur; le coup n'était pas à la mort, si la fortune ne l'eût rendu tel; et l'entreprise le tirer, étant à cheval et de loin, et à un qui se mouvait au branle de son cheval, fut l'entreprise d'un homme qui aimait mieux faillir son effet que faillir à se sauver ". On voit fort bien ici la différence de point de vue entre La Boétie et Montaigne. Le premier admire l'intention du tyrannicide, le second juge l'acte avec son auteur, au point de vue du courage. Les deux thèmes sont dans le poème de Torrentius. S'il a loué Gérard, ce n'est pas quoiqu'il fût un humaniste, c'est justement parce qu'il l'était. Il a suivi la mode d'un temps qui mit les assassins de César bien au-dessus de César lui-même.

Cette mode remonte aux débuts du siècle. Bien antérieure aux guerres de religion, elle vient en droite ligne de l'antiquité. Lucien a écrit un curieux plaidoyer intitulé le Tyrannicide. 
Voulant tuer un tyran, un homme tue le fils de celui-ci, sur quoi le tyran désespéré se détruit. Le meurtrier prétend avoir droit à la récompense promise au libérateur de l'État. Érasme et More ont donné tous deux une version de ce discours suivi d'une declamatio où ils rejettent la prétention du meurtrier tout en mettant l'accent sur la sainteté du tyrannicide $\left(^{(}\right)$:

Adeo imprudens non veretur pulcherrimum tyrannicidae titulum diis eripere, sibi erogare (Érasme).

Si tu interfecisses, nihil quererer, imo laudarem, admirarer, ac praemium primus decernerem (More).

Pour tous les deux, dans le cas présent, c'est la volonté des dieux qui a restitué la liberté de l'État.

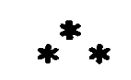

Lorsque P. Coste donna en 1724, à Londres, sa grande édition des Essais, il mit au passage cité ci-dessus la note que voici : "Balthasard Gérard, qui venait de tuer le prince d'Orange par un infâme assassinat ", sans paraître s'apercevoir combien cet adjectif détonne à côté du texte. Montaigne n'ignorait certes pas que Jauréguy, comme Gérard, s'introduisit auprès du prince en solliciteur, ce qui était assurément une trâitrise infâme, pour parler comme Coste. Son admiration pour l'assassin courageux n'en a pas été diminuée. Virtus, Virtù. Le poème de Torrentius, lui aussi, est d'un lecteur de Valère Maxime. 11 nous choque profondément. Montaigne n'y aurait rien trouvé à redire.

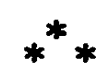

Lorsque Torrentius devint, en 1587, évêque d'Anvers, il eut à liquider une des séquelles de l'affaire Jauréguy. En même temps que celui-ci, en 1582 , on avait arrêté, puis mis à mort le dominicain Antoine Temmermann, qui semble n'avoir eu d'autre complicité avec Jauréguy que d'avoir connu son projet sous le sceau de la confession. Plusieurs magistrats

(1) Les Opuscula festivissima Luciani, qui contiennent ces quatre textes, imprimés pour la première fois chez Ascensius en 1506, ont été souvent réédités pendant la première moitié du siècle. 
anversois, catholiques in petto, mais collaborateurs plus ou moins contraints du gouvernement de Marnix, avaient dû prêter leur concours au procès de Temmermann. Le plus notable était Corneille Pruynen, trésorier d'Anvers et ami de Torrentius. Dès la prise de la ville par Farnèse, Pruynen et les autres s'occupèrent d'obtenir une absolution pour avoir participé au procès et au supplice d'un prêtre, affaire qui pouvait leur valoir de très graves ennuis. Les Jésuites la leur refusant, ils s'adressèrent au nouvel évêque.

Torrentius va-t-il les juger avec la même passion qu'il apporte à louer et Jauréguy et Gérard? Nullement. En bonne logique, cet enthousiaste du tyrannicide aurait dû accabler ceux qui l'avaient châtié. Il fait tout le contraire. Le 22 juill. 1586, de Liège où il est toujours, il écrit au nonce Bonhomini et lui demande les pouvoirs nécessaires pour absoudre les juges de Temmermann. Le nonce remet la décision à Sylvestre Pardo qui était vicaire-général sede vacante depuis l'année précédente, et requiert comme pénitence préalable une forte amende au bénéfice des Jésuites. Ceux-ci furent beaucoup plus difficiles à apaiser. Ils firent écrire à Torrentius par son vieil ami Baudouin Delange, recteur du collège de Louvain, une lettre très véhémente qui proteste contre l'indulgence de l'évêque à l'égard de deux "collaborateurs" notoires, Pruynen d'abord et ensuite Jean Vandernoot qui venait d'être choisi comme administrateur de St-Bernard alors que, abbé sous le régime calviniste, il avait aliéné d'importants biensfonds sans l'autorisation du pape. Nous n'avons pas la lettre de Delange, mais nous avons la réponse de Torrentius, du 15 févr. 1587 (1).

L'homme qui a écrit ces lignes est le contraire d'un fanatique. C'est, tout au rebours, le politique qui avait pour principe: Remedium malorum omnium optimum oblivio praeteritorum est.

Tant de textes rapprochés ne sont peut-être pas de trop pour donner à sa Laus Baltasaris Gerardi fortissimi tyrannicidae son éclairage véritable.

Marie Delcourt.

(1) Corresp. de Torrentius, T. I, L. 205, 212, $218,254$. 